\title{
The Commercial Analysis of Three Space Debris Mitigation Schemes
}

\author{
Jiali Chen \\ NORTH CHINA ELECTRIC POWER UNIVERSITY Baoding \\ Jialichen6666@163.com
}

Keywords: Space Debris, Economic Indicator, Analytic Hierarchy Process, Synthesis Score.

\begin{abstract}
This paper firstly introduces three common space debris mitigation schemes including laser removal, satellite removal and combination method. Then the evaluation model is built to estimate the three space debris mitigation schemes based on the Analytic Hierarchy Process. Finally, it is divided into six different scenarios in terms of space debris's size and assembles density.
\end{abstract}

\section{Introduction}

Since the number of satellites in earth orbit is steadily increasing, space debris, if left unchecked, will eventually pose a serious hazard to near-Earth space activities, so effective measures should be taken to mitigate it.[1] There are some previous studies analyzed space debris removal in the aspect of technology and economy. Many researchers explored the developing of space debris nowadays, for example, J.C. Dolado-Perez presented and discussed many of the uncertainty sources affecting the long-term predictions done with evolutionary models [2]. So far, there are mainly three effective schemes to remove space debris including laser removal, satellite removal and combination scheme.[3]

\section{2、 Different Scenarios Analyzing Model}

\section{1、 Analytic Hierarchy Process method}

The greatest eigenvalue of matrix $\mathrm{A}$ is $\lambda_{\max }$, and the corresponding eigenvector is $u=\left[\begin{array}{lllll}u_{1} & u_{2} & u_{3} & \ldots & u_{n}\end{array}\right]$, then we normalize the $\mathrm{u}$ by the expression:

$$
x_{i}=\frac{u_{i}}{\sum_{i=0}^{n} u_{j}}
$$

Do the consistency check

The indicator of consistency checks formula:

$$
C I=\frac{\lambda_{\max }-n}{n-1}
$$

Where $n$ denotes the exponent number of matrix,

The expression of consistency ratio:

$$
C R=\frac{C R}{R I}
$$

$C W_{i}$ denotes the weight of $i^{\text {th }}$, criteria level factor, where $A W_{j}$ is the weight of $j^{\text {th }}$, secondary critical level factor, and $F_{j}$ denotes the $j^{\text {th }}$ secondary critical level factor.

The evaluation grade $\mathrm{Y}_{1}$ is should be:

$$
Y_{1}=\sum_{i=1}^{5} c W_{i} * \sum_{j=1}^{m i} A W_{j} * F_{j}
$$

\subsection{Synthesis Score}

The synthesis score can be determined by the Analytic Hierarchy Process method. We establish a judgment matrix $J$ as follows: 


$$
J=\left[\begin{array}{cccc}
1 & \frac{1}{3} & \frac{1}{7} & \frac{1}{5} \\
3 & 1 & 3 & 5 \\
7 & \frac{1}{3} & 1 & 3 \\
5 & \frac{1}{5} & \frac{1}{3} & 1
\end{array}\right]
$$

Then determine the weight of four economic indicators:

The feature vector $W$ of the judgment matrix $J$ with respect to the maximum eigenvalue, which is also the relative importance sort weight of the same layer to the former layer, is the hierarchical single sort.

So the weight of four economic indicators is:

$$
\left[\begin{array}{llll}
w_{R} & w_{C} & w_{B} & w_{I}
\end{array}\right]=\left[\begin{array}{llll}
0.0654 & 0.4972 & 0.2926 & 0.1449
\end{array}\right]
$$

Where $w_{i}$ is the weight of risk, $w_{C}$ is the weight of cost, $w_{B}$ is the weight of benefits and $w_{I}$ is the weight of interference.

Assume that $W_{i}$ is composite indicator of one of the removal methods and we derive the equation as

$$
W_{i}=R_{i} w_{R}+C_{i} w_{c}+B_{I} w_{B}+I_{i} w_{I}
$$

Where $R_{i}$ is the indicator of risk, $B_{b}$ is the indicator of benefits, $I_{i}$ is the indicator of interference and $C_{i}$ is the indicator of cost which can expressed as follows.

$$
C_{i}=\left\{\begin{array}{l}
0.015 n_{1}+1.05, i=1 \\
0.020 n_{2}+0.75, i=2 \\
0.015 \varepsilon+0.020(1-\varepsilon)+0.9, i=3
\end{array}\right.
$$

\subsection{The Evaluation of Space Debris Removal Methods}

Index $b$ is the proportion of $n_{1}$ in $n$, that is to say, $b=n_{1} /\left(n_{1}+n_{2}\right)$

The cost is proportional to $W_{i}$, besides, the benefit and interference is fixed when the size of space debris is settled.

\subsubsection{Situation I}

When the size of space debris is variable,
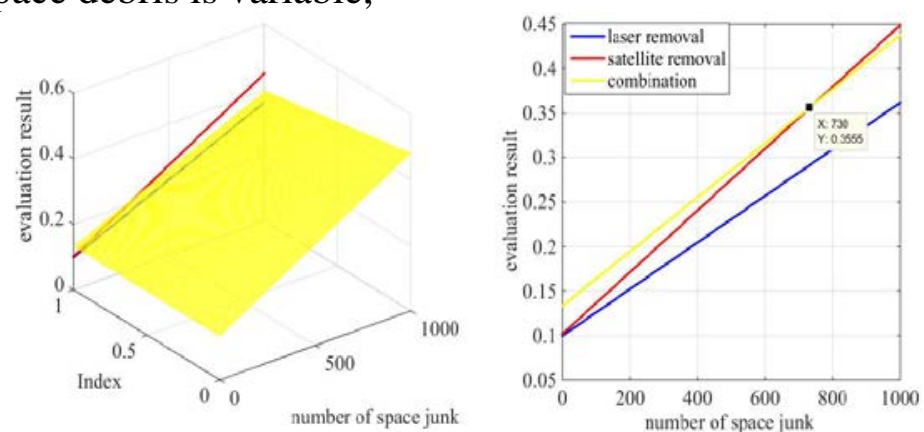

Fig. 1 low collision probabilities in situation I

As is described in Fig.5, the left figure is the evaluation result when index b ranges from 0 to 1 , while the right figure is the evaluation result when index $b$ is a fixed number (we assume $b$ is 0.5 ).

According to Fig.5, when the space debris is a mixture of small debris and medium debris, the optimal scheme is laser removal. That is because the risk of new debris generation is significantly high using laser system. 

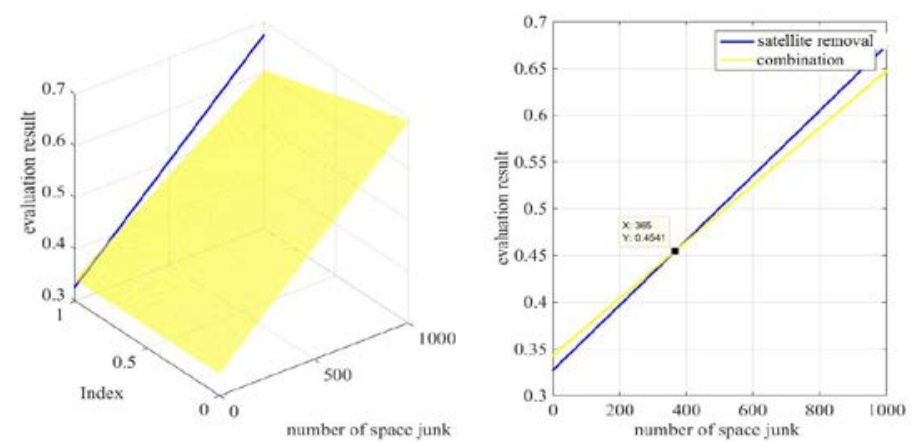

Fig. 2 medium collision probabilities in situation I

As is shown in Fig.6, when the space debris is a mixture of small and large debris or medium and large debris, the optimal scheme is depends on circumstances. If the number of space debris is large, the optimal scheme is combination; if the number of space debris is little, we choose laser removal as the best scheme. That is because laser system is not available for big space debris removal. When dealing with big debris, we had better use the combination method.
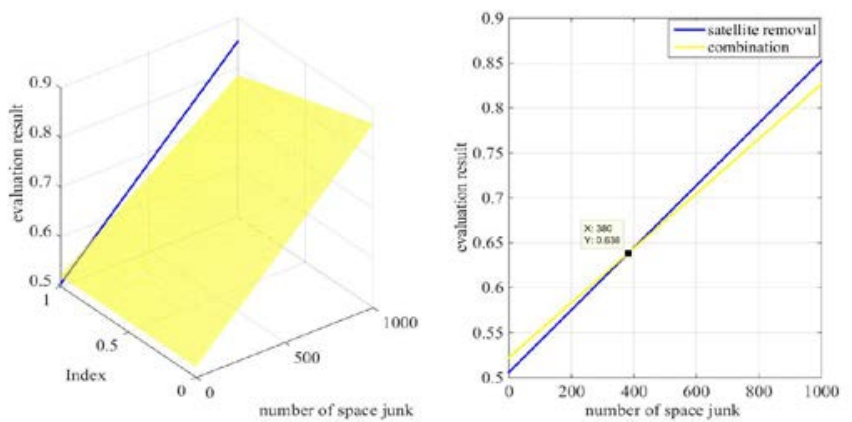

Fig. 3 high collision probabilities in situation I

As is indicated in Fig.7, when the space debris is a mixture of all kind size of debris, the best scheme is similar to medium collision probabilities.

\subsubsection{Situation II}

When the size of space debris is single,
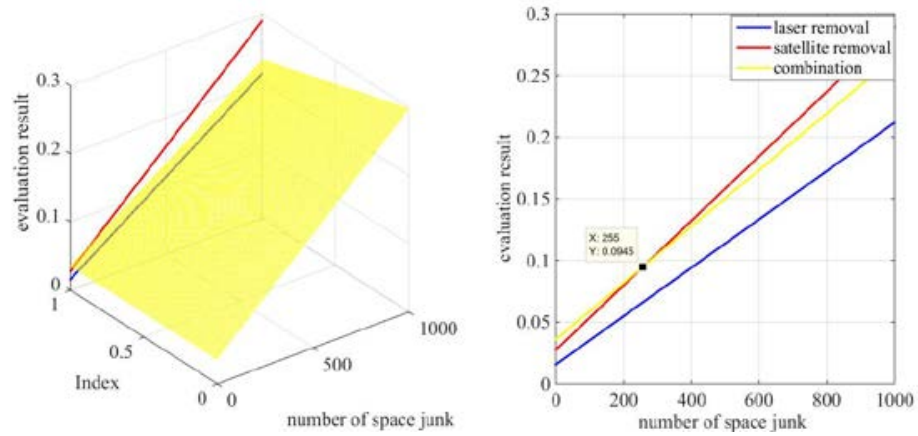

Fig. 4 low collision probabilities in situation II

When the space debris is small debris, the optimal scheme is laser removal.
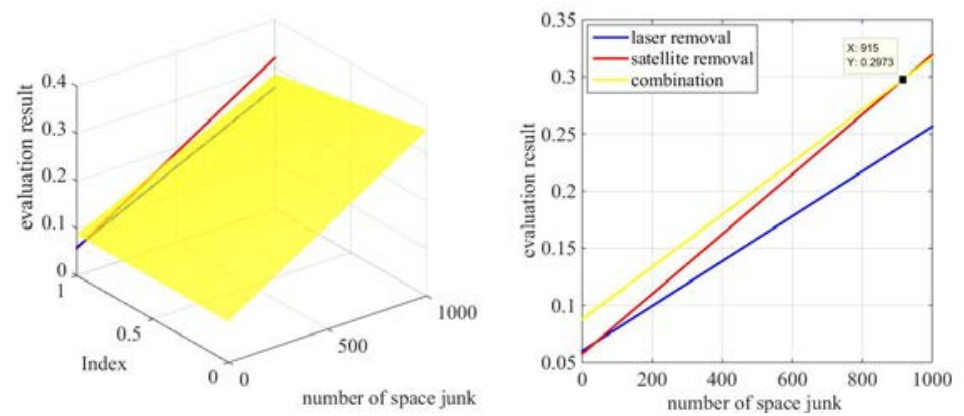

Fig. 5 medium collision probabilities in situation II

When the space debris is medium debris, the optimal scheme is depends on the number of space 
debris, If the number of space debris is very small, the optimal scheme is combination method; if the number of space debris is large, we choose satellite removal as the best scheme.
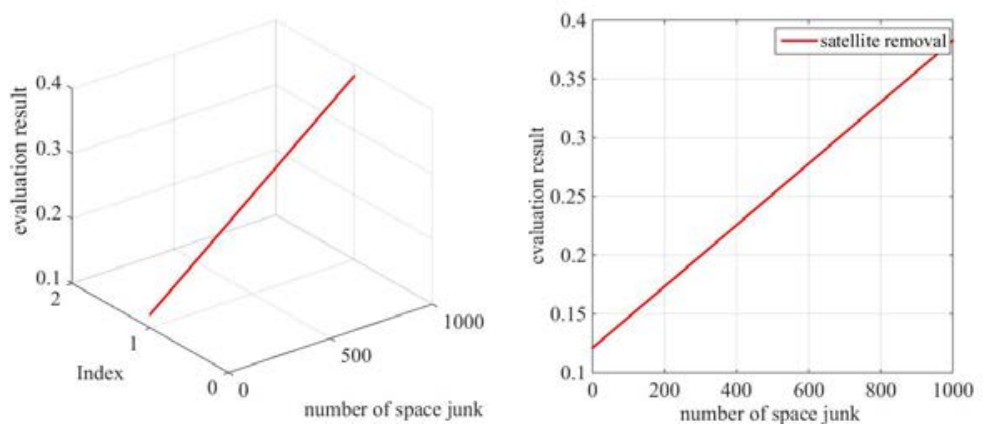

Fig. 6 high collision probabilities in situation II

When the space debris is big debris, the optimal scheme is satellite removal.

\section{Summary}

Above all, it can be drew the conclusions as follows:

[1] small assemble density, big size: satellite removal

[2] small assemble density, medium size: satellite removal or laser removal

[3] small assemble density, small size: laser removal

[4] big assemble density, small and medium size: laser removal

[5] big assemble density, small and large debris or medium and large debris: satellite removal or combination

[6] big assemble density, small, medium and large debris: satellite removal or combination

\section{Reference}

[1] Venturelli, P.; Velasco, A.M., "A method for addressing the danger of space debris," in Aerospace Conference, 2011 IEEE , vol., no., pp.1-10, 5-12 March 2011

[2] J.C. Dolado-Perez, Carmen Pardini, Luciano Anselmo, Review of uncertainty sources affecting the long-term predictions of space debris evolutionary models, Acta Astronautica, Volume 113, August-September 2015, Pages 51-65, ISSN 0094-5765, http://dx.doi.org/10.1016/j.actaastro.2015.03.033.

[3] Minghe Shan, Jian Guo, Eberhard Gill, Review and comparison of active space debris capturing and removal methods, Progress in Aerospace Sciences, Volume 80, January 2016, Pages 18-32, ISSN 0376-0421, http://dx.doi.org/10.1016/j.paerosci.2015.11.001. 